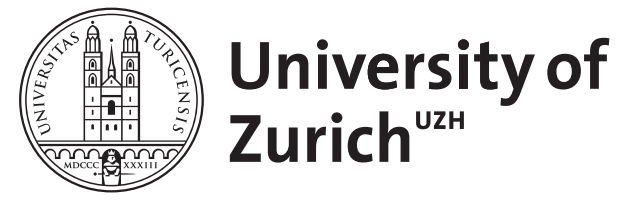

\title{
Do the diagnostic criteria differ between alcoholic and nonalcoholic chronic
} pancreatitis?

Ammann, Rudolf W ; Mullhaupt, Beat

DOI: https://doi.org/10.1007/s00535-006-1940-9

Posted at the Zurich Open Repository and Archive, University of Zurich ZORA URL: https://doi.org/10.5167/uzh-156610

Journal Article

Published Version

Originally published at:

Ammann, Rudolf W; Mullhaupt, Beat (2007). Do the diagnostic criteria differ between alcoholic and nonalcoholic chronic pancreatitis? Journal of Gastroenterology, 42(S17):118-126.

DOI: https://doi.org/10.1007/s00535-006-1940-9 


\title{
Do the diagnostic criteria differ between alcoholic and nonalcoholic chronic pancreatitis?
}

\author{
Rudolf W. Ammann and Beat Mullhaupt \\ Division of Gastroenterology and Hepatology, and Swiss HPP Center University Hospital Zurich, CH-8091 Zurich, Switzerland
}

Key words: alcoholic chronic pancreatitis, idiopathic juvenile early onset chronic pancreatitis, idiopathic senile late onset chronic pancreatitis

Chronic pancreatitis $(\mathrm{CP})$ is a progressive inflammatory process, which usually evolves over years into pancreatic cirrhosis, irrespective of endoscopic or surgical interventions, except autoimmune pancreatitis (reversed by steroids) or obstructive pancreatitis (relieved by removal of obstruction). The latter two conditions are not considered here, particularly because the typical progressive evolution of CP is lacking.

In the diagnosis of CP two major problems need special attention: (1) staging, that is, differentiation between early- versus late-stage $\mathrm{CP}$ and (2) etiology, specifically the impact of etiology on the evolution of $\mathrm{CP}$ from disease onset . Our presentation is focused primarily on these aspects and is based in particular on data from our prospective long-term study.

\section{Staging of CP}

$\mathrm{CP}$ evolves over years and is diagnosed reliably in advanced stages in accordance with the diagnostic hallmarks: pancreatic calcification, marked ductal alterations (Cambridge criteria), and irreversible exocrine (endocrine) insufficiency. Diagnosis of late-stage CP is identical for both alcoholic and nonalcoholic CP.

\section{Diagnostic challenge of early-stage $\mathbf{C P}$}

The diagnosis of early-stage $\mathrm{CP}$-in contrast to advanced late-stage $\mathrm{CP}$-is problematic (Fig. 1). Three major variables are important markers of the disease

Reprint requests to: $\mathrm{B}$. Mullhaupt course from onset to late-stage $\mathrm{CP}$ : clinical features, morphology, and function. Clinical features and function are closely interrelated variables and change in association with progressive acinar destruction and fibrosis from disease onset to late-stage CP.

Early-stage CP usually starts with recurrent acute pancreatitis. The diagnostic criteria of acute pancreatitis are well established. The broad overlap of clinical features in acute pancreatitis irrespective of etiology, in particular between gallstone and alcoholic pancreatitis, the two most prominent causes of acute pancreatitis in industrialized countries $(60 \%-80 \%)$, renders a precise differentiation between acute and chronic pancreatitis virtually impossible at onset. Many experts, therefore, prefer to label initial episodes of acute pancreatitis according to etiology, for example, as alcoholic or gallstone pancreatitis.

Alcohol abuse is a prominent risk factor for both acute and chronic pancreatitis. The debate on classification of CP has been confounded during the past 40 years by the dogma of Marseille, which postulates that (1) acute pancreatitis and $\mathrm{CP}$ are two distinct nosological entities and (2) acute pancreatitis virtually never progresses to $\mathrm{CP}$. In fact, gallstone pancreatitis rarely progresses to $\mathrm{CP} .{ }^{1}$

In contrast, most experts currently agree that alcoholic and hereditary $\mathrm{CP}$ are the end result of repeated episodes of acute pancreatitis, that is, the necrosis-fibrosis hypothesis of CP by Klöppel and Maillet. $^{2}$

Basically, there are three major challenges in the diagnosis of early-stage CP: (1) pancreatic histology, which is the gold standard of early-stage $\mathrm{CP}$, is rarely available; (2) clinical, functional, and morphological features are identical at onset in both acute reversible (e.g., gallstone) and progressive (alcoholic) pancreatitis; and (3) a time lag, averaging $4.5-5.5$ years, exists between acute onset pancreatitis and documentation of markers of advanced alcoholic CP. ${ }^{3-5}$ 


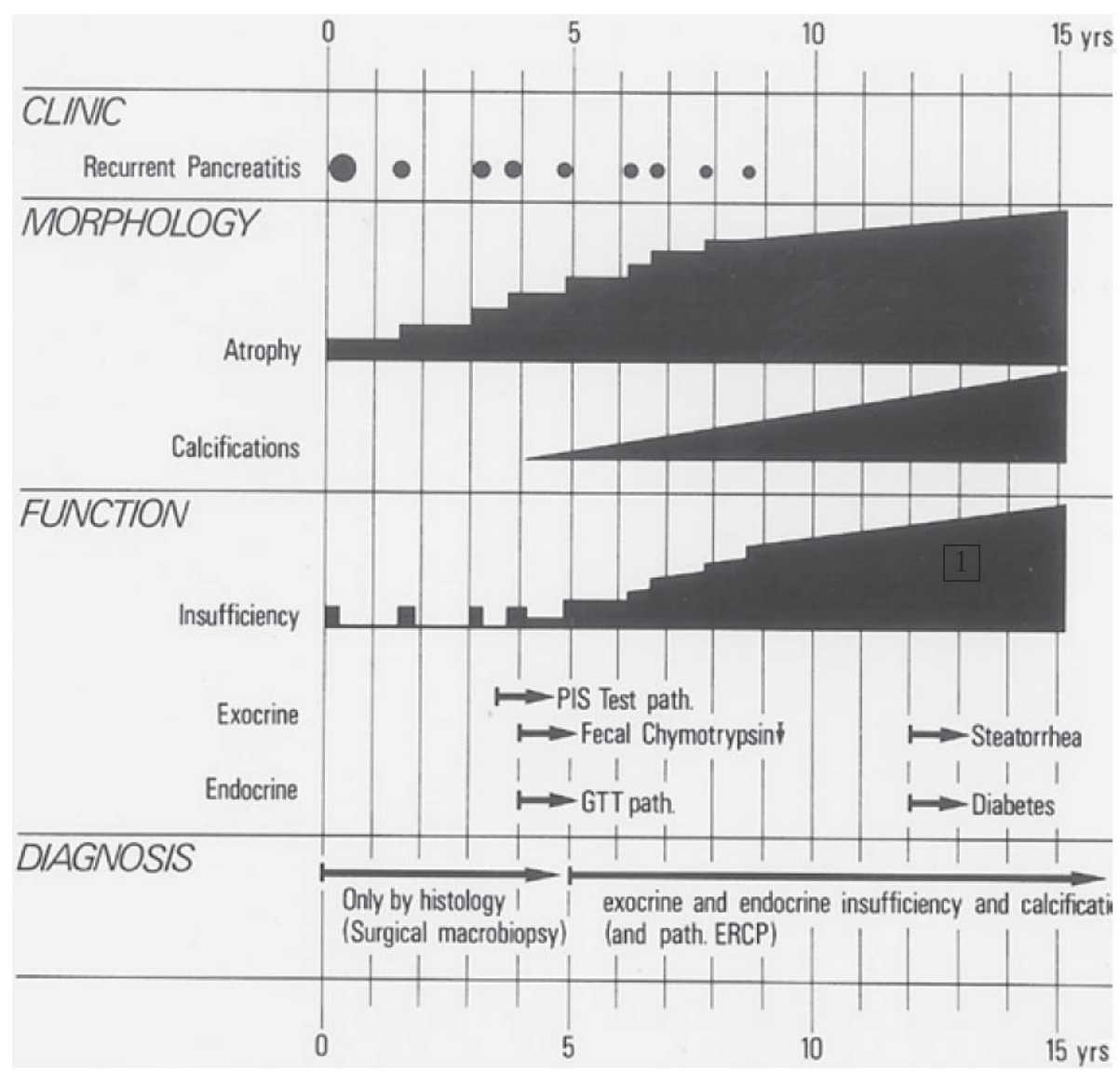

Fig. 1. Scheme of the long-term evolution of the three major, closely interrelated variables that change in association with progressive acinar atrophy/fibrosis after onset of chronic pancreatitis (CP). For instance, acute pancreatitis is the prominent clinical feature of the early-stage disease, while the markers of late-stage $\mathrm{CP}$ (persistent exocrine insufficiency, diabetes, calcification) develop after a delay of several years (time lag). The diagnostic gold standard of early-stage $\mathrm{CP}$ is pancreatic histology (rarely available). Acute-onset pancreatitis and the signs of late-stage $\mathrm{CP}$ are the two cornerstones of $\mathrm{CP}$ in the absence of histology. CP can be suspected in cases of recurrent alcoholic pancreatitis (probable CP). Definite $\mathrm{CP}$ is, however, best proven (or excluded) by documentation of signs of late-stage CP (see Figs. 3-5). $P / S$, pancreozymin-secretin; $G T T$, glucose tolerance test; $E R C P$, endoscopic retrograde cholangiopancreatography

\section{Time lag between acute onset pancreatitis and definite (advanced) CP}

Monitoring of pancreatic function is important for differentiating acute reversible and chronic progressive pancreatitis in the early stages of disease if pancreatic histology is not available. For instance, the fecal chymotrypsin profile over a period of more than 10 years from disease onset is documented in a series of acute (gallstone) pancreatitis attacks $(n=74)$ versus alcoholic CP $(n=114)$ (Fig. 2). Obviously, the fecal chymotrypsin profile remained normal $(>120 \mu \mathrm{g} / \mathrm{g})$ in acute pancreatitis for $>10$ years. In contrast, the fecal chymotrypsin profile became progressively pathological in CP. However, the first definitely low values occurred 3 years after onset. In other words, there was a typical time lag-a diagnostic "black-box" period-between disease onset and the development of markers of advanced late-stage CP. ${ }^{3-5}$

Thus, a definite categorization of pancreatitis in its initial stages is often impossible because of this time lag. The following case reports illustrate the problem in a few patients with acute onset pancreatitis.

\section{Case 1.60-year-old woman}

This patient had biliary acute pancreatitis combined with obstructive cholestasis due to choledocholithiasis. She initially showed mild pancreatic insufficiency, as proven by a pathological pancreozymin-secretin test (P/S-Test) and low fecal chymotrypsin values (FCT) over an 8-month period after disease onset (Fig. 3). Some experts would probably conclude that the patient has CP. However, the patient was followed-up for 21 months. At 10 months, a second P/S-Test was normal, and the FCT profile normalized by 21 months after disease onset. Thus, the final interpretation of case 1 was acute biliary pancreatitis with transient (reversible) pancreatic insufficiency.

\section{Case 2. 41-year-old man}

The patient had a first severe episode of acute alcoholic pancreatitis in 1974. In 1975, a second mild attack followed. Thereafter, the patient remained asymptomatic despite continuation of alcohol consumption in moderate amounts. Yearly control studies documented no evidence of exocrine insufficiency for 12 years after disease onset, with constantly high-normal FCT values 


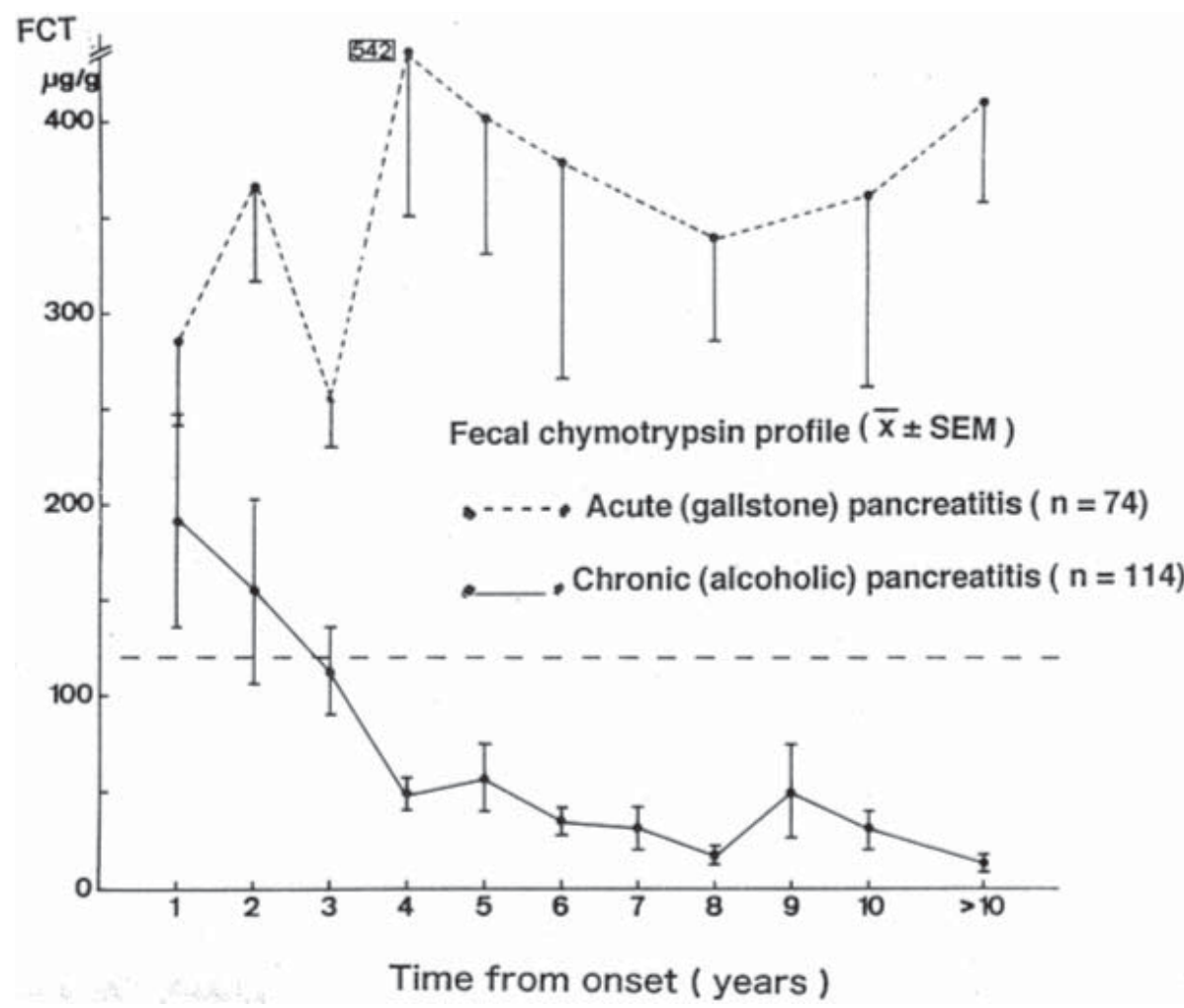

Fig. 2. Fecal chymotrypsin profile over a period of more than 10 years from onset of acute gallstone $(n=74)$ versus chronic alcoholic pancreatitis $(n=114)$ (Mean \pm SEM). In alcoholic $\mathrm{CP}$, the fecal chymotrypsin values progressively drop, but they become abnormal $(<120 \mu \mathrm{g} / \mathrm{g})$ only after a time lag of 3-4 years after disease onset. (Note that the fecal elastase I test was not available before 1995) female, 60 ys old

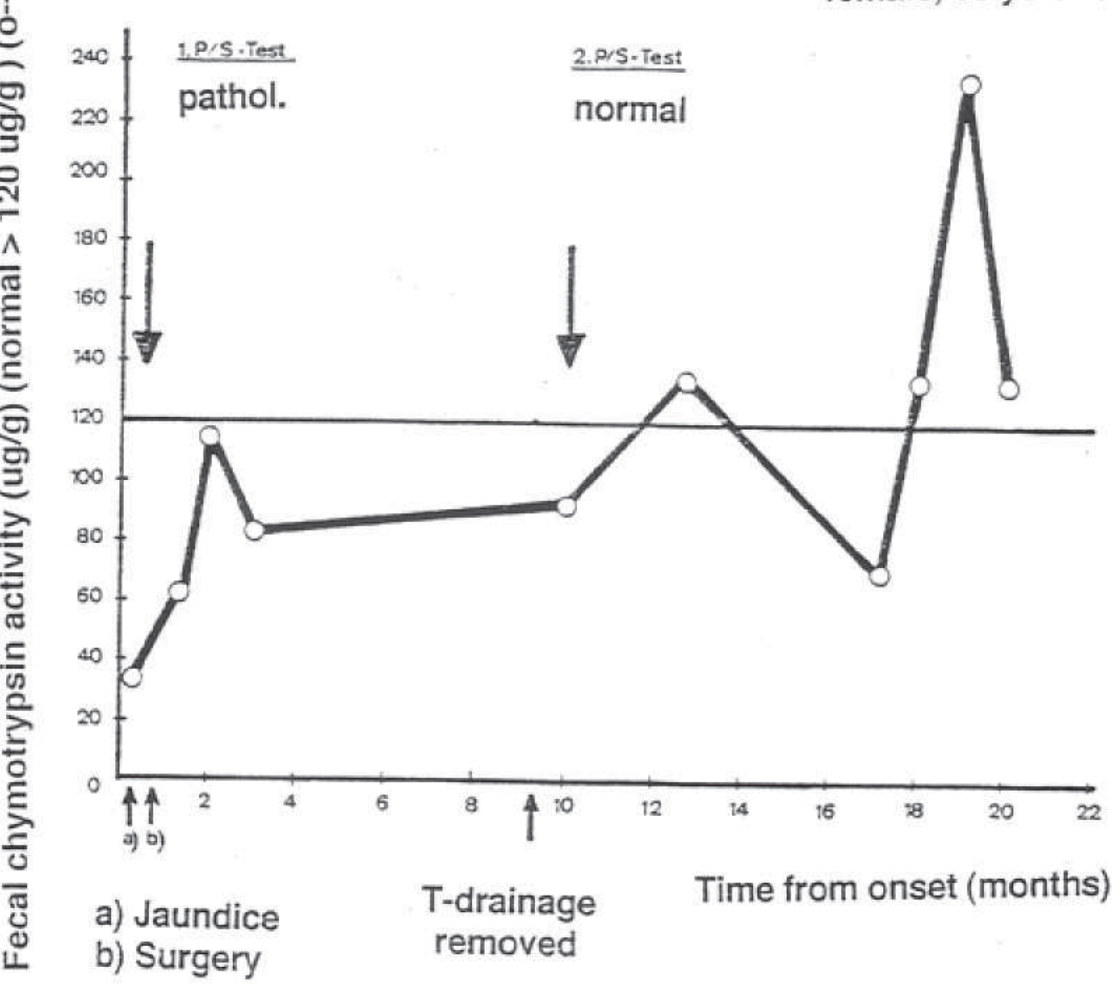

Fig. 3. Case 1. This female patient developed exocrine insufficiency following acute gallstone pancreatitis (fecal chymotrypsin concentration $<120 \mu \mathrm{g} / \mathrm{g}$; P/S-Test) that lasted 8 months from onset, suggesting chronic pancreatitis. However, the $\mathrm{P} / \mathrm{S}$ test results were normal at 10 months, and the fecal chymotrypsin level was normal at 21 months after disease onset, indicating that the exocrine insufficiency was reversible and that progression to $\mathrm{CP}$ is unlikely in this patient 
(Fig. 4). Some experts would probably exclude CP in this patient. However, 13 years after onset, the FCT values suddenly dropped to very low levels and calcification was documented for the first time. Thus, the final interpretation in case 2 was alcoholic CP, diagnosed after a time lag of 13 years after disease onset.
Case 3. 34-year-old man

This alcoholic patient had four episodes of acute pancreatitis within 1 year from onset. The FCT profile normalized at 1.5 years after onset and remained normal until 8 years after onset, probably owing to cessation of alcohol

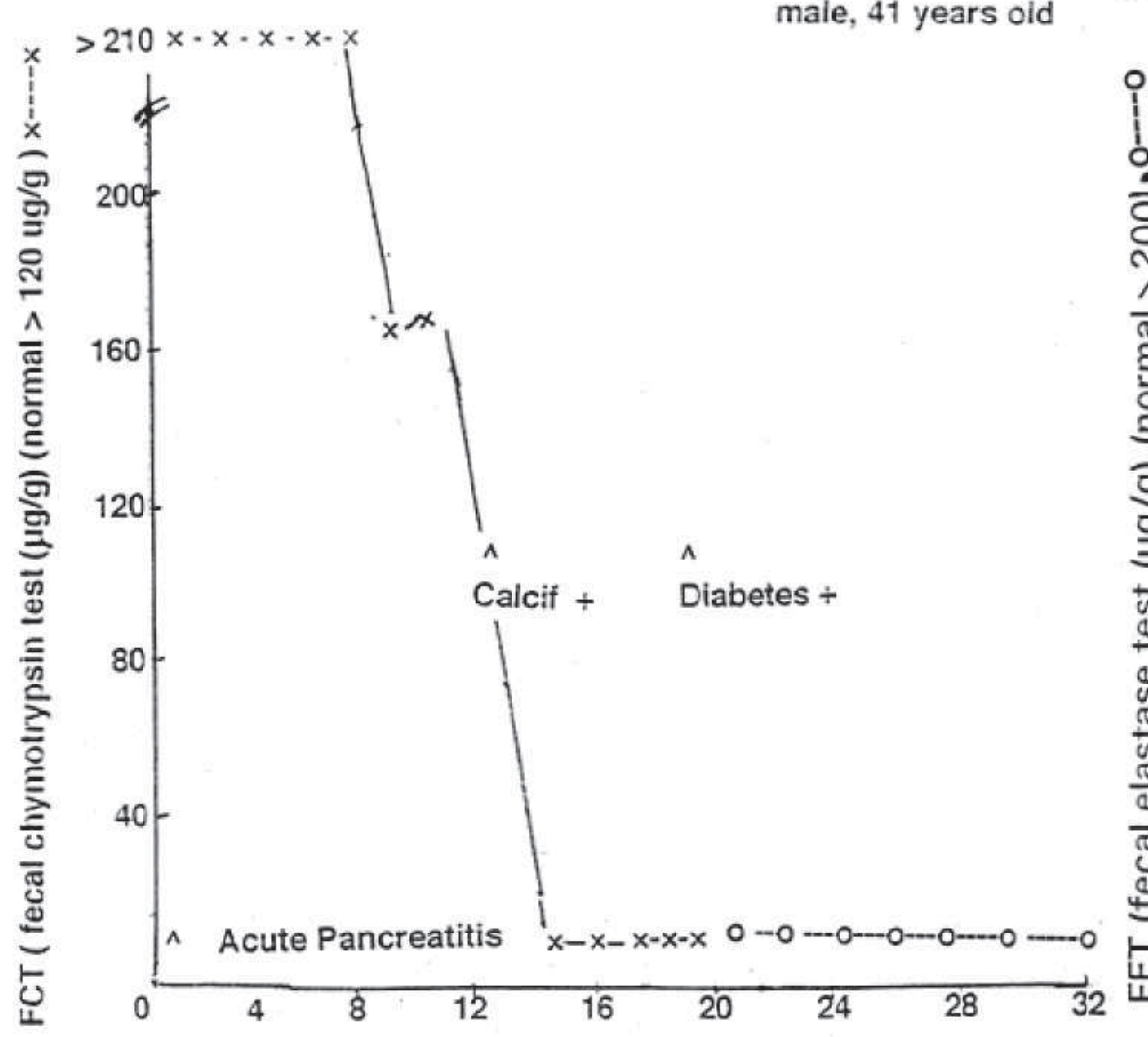

Time from onset ( years )

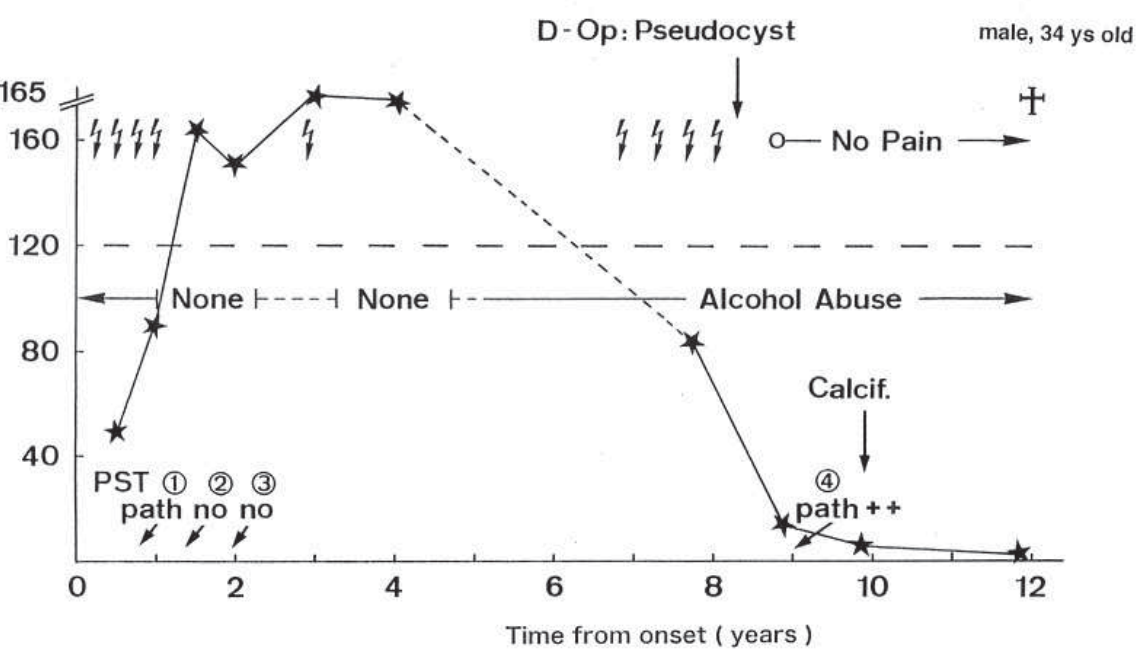

Fig. 4. Case 2. Long-term disease evolution in a patient with severe acute alcoholic pancreatitis in 1974, followed by a second mild pancreatitis attack in 1975. Thereafter, the patient remained asymptomatic despite continued reduced alcohol intake (i.e., the pain probably stopped because of the alcohol reduction). No evidence of exocrine insufficiency was found at yearly control studies for 12 years after onset. After this time lag, the fecal chymotrypsin values suddenly dropped, calcification was noted, and diabetes (controlled by oral antidiabetics) developed at 20 years after onset

Fig. 5. Case 3. Long-term disease evolution following four episodes of acute alcoholic pancreatitis within 1 year. Exocrine insufficiency (path) was documented by the first P/S-Test $(P S T)$, and low fecal chymotrypsin values reverted to normal 1.5 years after onset, probably due to cessation of alcohol intake. At 7 years after onset, pancreatitis recurred, and persistent exocrine insufficiency developed following a time lag of 8 years after onset, in association with pancreatic calcification (Calcif.) at 10 years after onset. Despite continued alcohol abuse, no further episodes of pancreatitis occurred in the last 4 years of life (burn-out of late-stage $\mathrm{CP}$; see text below). D-Op, Drainage operation 
abuse (Fig. 5). Some experts would probably conclude that progression to $\mathrm{CP}$ is unlikely in this patient. However, between 7 and 8 years after onset, pancreatitis recurred: a pancreatic pseudocyst developed, which was treated by a surgical drainage procedure. Exocrine insufficiency developed at 8 years after onset (low FCT, pathological P/S-Test). Calcification was documented at 10 years after onset. Thus, the final interpretation in case 3 was alcoholic chronic pancreatitis, first documented after a time lag of 9 years after onset of disease.

\section{Preliminary conclusions}

1. In patients with first onset pancreatitis, categorization as acute versus chronic pancreatitis is rarely possible without histology.

2. Prolonged surveillance of patients is necessary to prove or exclude $\mathrm{CP}$ because of the delay in development of markers of CP (time lag).
3. It is likely that a large percentage of patients classified as having acute alcoholic pancreatitis will progress to $\mathrm{CP}$ if followed up for years.

\section{Rate of progression of $\mathrm{CP}$}

The rate of progression to alcoholic late-stage CP from disease onset varies considerably (Table 1), but the reasons for this variable rate are unknown. There is, however, evidence that cessation or a reduction of alcohol abuse may delay the progression to advanced CP. ${ }^{8}$ Moreover, smoking appears to accelerate the progression to calcification, as shown by a recent international multicenter study ${ }^{9}$ (Fig. 6). Etiology is an additional factor that impacts the progression rate of $\mathrm{CP}$ (see below).

There are only limited data on the relationship between morphology and function in the course of the disease from onset to late-stage CP. Our group studied this

Table 1. Rate of progression to and incidence of late-stage alcoholic chronic pancreatitis

\begin{tabular}{lcc}
\hline & $\begin{array}{c}\text { Mayo Clinic } \\
(n=249)^{6}\end{array}$ & $\begin{array}{c}\text { Zurich series } \\
(n=265)^{7}\end{array}$ \\
\hline Calcification & 59 & 86 \\
$\quad$ Incidence (\%) & 8.7 & $6(0-18)$ \\
Median time after onset (years) (range) & 48 & 96 \\
Exocrine insufficiency & 13.1 & $5(1-25)$ \\
$\quad$ Incidence (\%) & 38 & 66 \\
Median time after onset (years) (range) & 19.8 & $8(1-25)$ \\
Diabetes & & \\
Incidence (\%) & & \\
Median time after onset (years) (range) & & \\
\hline
\end{tabular}

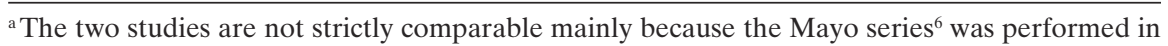
part retrospectively
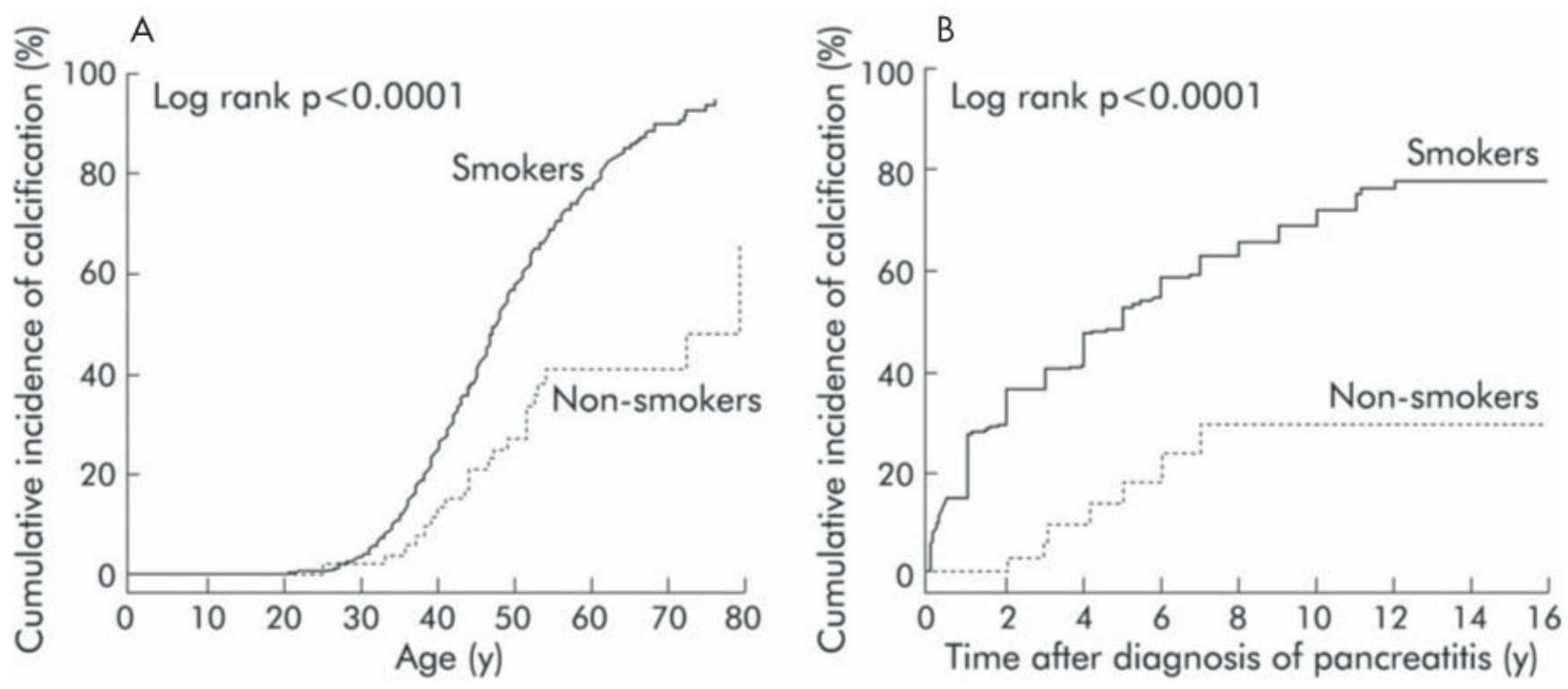

Fig. 6. Rate of progression to pancreatic calcification is significantly increased by smoking in alcoholic $\mathrm{CP}^{9}$ 
issue in 73 alcoholic CP patients using either surgical or necropsy specimens. Surgical and necropsy specimens were available in 10 of 73 patients (Figs. 7 and 8). ${ }^{10}$

The median fibrosis score (FS, on a scale of 0-12) increased from 6.2 within 4 years of onset to 10.2 at $\geq 13$ years after onset. Calcification (\%) increased almost threefold between onset and $\geq 13$ years after onset, closely matching the increase in fibrosis (Fig. 7).

Exocrine insufficiency (fecal chymotrypsin $<120 \mathrm{mg}$ / g) was closely correlated with the fibrosis score and was noted in $87 \%-97 \%$ of patients with a fibrosis score of 7 to 12 (Fig. 8). Diabetes was observed in about $60 \%$ of patients with a fibrosis score of $\geq 10$ but in only about $15 \%$ of those with a fibrosis score of $\leq 9$.

These findings indicate that repeated function testing is a rather reliable surrogate for histology for monitoring the progression of CP. Moreover, the data support the notion that advanced late-stage $\mathrm{CP}$ can be diagnosed reliably by most available routine methods if adequate histological specimens are not available.

By applying strict criteria, a diagnosis of $\mathrm{CP}$ has been confirmed at our center over the last 40 years in a total of 343 patients. $^{7}$ At the same time, an equal number of patients $(n=385)$ with suspected but unproven CP (socalled probable $\mathrm{CP}$ ) were excluded because histology or data indicating progression to late-stage $\mathrm{CP}$ were lacking.

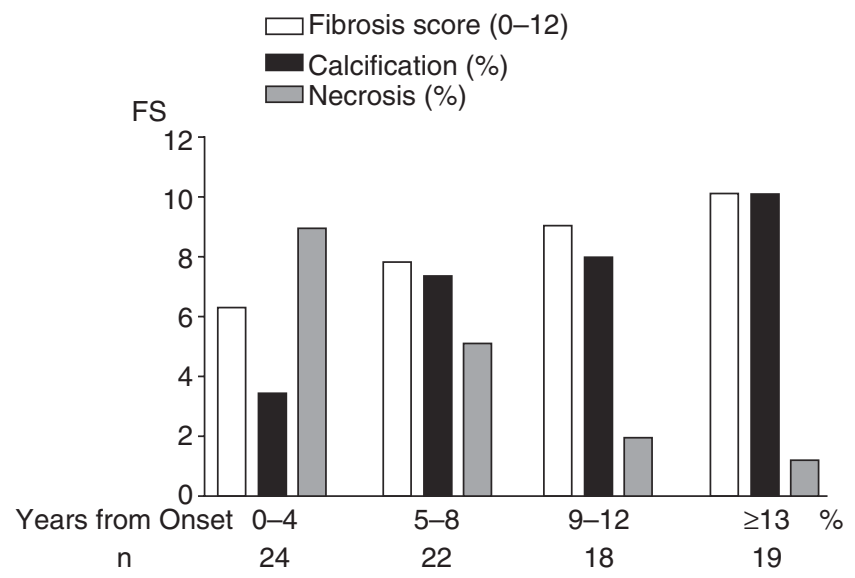

Fig. 7. Correlation of the fibrosis score (FS; histology score $0-12$ ) in relation to duration of $\mathrm{CP}$ in a series of 73 consecutive patients with alcoholic CP. Large pancreas specimens (biopsy or autopsy specimens, at different time intervals after onset), were assessed by two experienced pathologists according to the scoring system. The median FS almost doubled from 6.2 (at 4 years after onset) to 10.2 (at $\geq 13$ years after onset). Calcification increased about threefold, in close relation with the FS (modified data from reference 10)

\section{Impact of etiology on the rate of progression of CP}

Knowledge of the natural history of $\mathrm{CP}$, which is the backbone of CP diagnosis, is still limited. For instance, only a few previous prospective studies have investigated the long-term evolution of alcoholic versus nonalcoholic CP separately. ${ }^{7}$ At present, the assumption prevails that the natural history of $\mathrm{CP}$ is identical irrespective of etiology. ${ }^{11}$

Diagnosis of advanced CP may be delayed by many years in patients with slow CP progression, as shown above. Interestingly, the progression rate is particularly delayed in patients with nonalcoholic $\mathrm{CP}$, as shown by our group ${ }^{7}$ and by a Mayo Clinic series. ${ }^{6}$

Idiopathic juvenile "early onset" CP (IJCP), idiopathic senile "late onset" $\mathrm{CP}$ (ISCP), and hereditary CP (HP) are three major distinct nosological entities of nonalcoholic $\mathrm{CP}$ that differ with respect to age at onset, clinical features ${ }^{6,7,9,10}$ (Table 2), and presence of gene mutation. ${ }^{12,13}$ The final pathway of alcoholic and nonalcoholic CP is almost identical at late stages, as documented by the high incidence of calcification and exocrine insufficiency (Table 2).

Etiology has a major impact on the progression rate of $\mathrm{CP}$. In particular, early-stage $\mathrm{CP}$ lasts significantly longer in IJCP and HP than in alcoholic CP (ACP). ${ }^{7}$ The development of exocrine insufficiency after disease onset is significantly delayed in IJCP and HP versus ACP (Fig. 9). Documentation of calcification is also markedly delayed after disease onset in HP and IJCP versus ACP (without reaching significance) (Fig. 10). In ISCP, constituting a large proportion of primary painless CP (i.e., unknown onset),

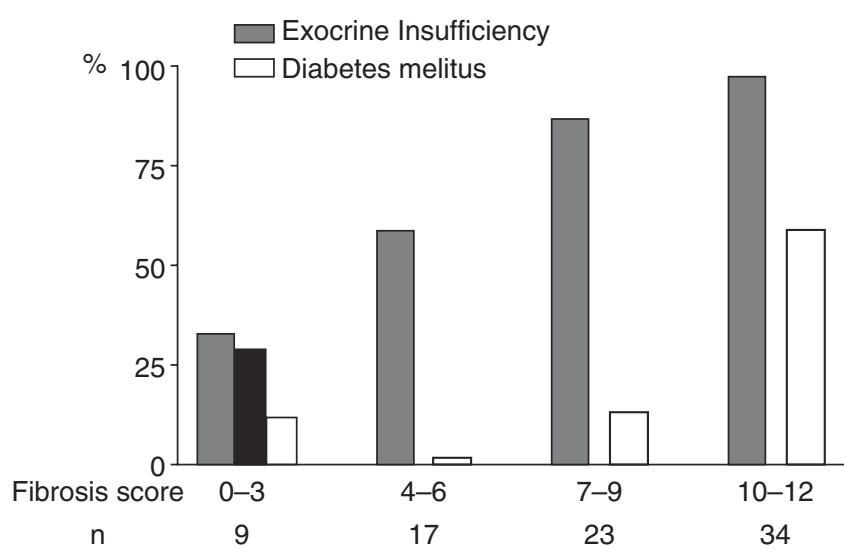

Fig. 8. Relationship between the fibrosis score and pancreatic dysfunction (see Fig. 7). Exocrine insufficiency was noted in $87 \%-97 \%$ of patients with a fibrosis score of $7-12$. Diabetes was noted in about $60 \%$ of patients with a fibrosis score of $\geq 10$ but in only $13 \%$ of those with a fibrosis score of $\leq 9$ (modified data from reference 10) 
pancreatic insufficiency or pancreatic calcification were present in up to $70 \%$ of patients at the time of diagnosis of CP. The development of diabetes differed also significantly in HP and IJCP compared with ACP (Fig. 11). In ISCP, diabetes was present in $50 \%$ of patients with primary painless $\mathrm{CP}$ at diagnosis.

Obviously, our series is small and additional prospective long-term studies of mixed medical-surgical CP series are required to corroborate our findings on the impact of etiology on the long-term evolution of CP. On the other hand, our data are in agreement with the results of the Mayo Clinic series (except for HP, which was not considered in that series) ${ }^{6}$ and with data of a large European multicenter study of HP. ${ }^{14}$
Of special interest in this regard is the debated issue of the relationship between exocrine insufficiency and the pain profile in CP. ${ }^{7,15}$ According to the experience of our group and others, ${ }^{7,16,17}$ lasting pain relief occurs in uncomplicated late-stage $\mathrm{CP}$ in association with progressive exocrine insufficiency-burn-out of $\mathrm{CP}$-both in alcoholic and nonalcoholic CP. Because of the delay in the progression, the precalcific painful early stage lasts considerably longer in IJCP and HP than in ACP. ${ }^{7}$ This observation emphasizes the importance of staging $\mathrm{CP}$, which depends largely on function testing, a currently often-dismissed issue in CP diagnosis and therapy. ${ }^{15-18}$ This controversy, which has yet to be settled, is beyond the scope of the present review.

Table 2. Clinical characteristics of alcoholic versus nonalcoholic CP (idiopathic juvenile, idiopathic senile, and hereditary $\mathrm{CP}$ )

\begin{tabular}{lcccc}
\hline & $\begin{array}{c}\text { ACP } \\
(n=265)\end{array}$ & $\begin{array}{c}\text { IJCP } \\
(n=21)\end{array}$ & $\begin{array}{c}\text { ISCP } \\
(n=46)\end{array}$ & $\begin{array}{c}\text { HP } \\
(n=11)\end{array}$ \\
\hline Male (\%) & 90 & 71 & 87 & 46 \\
Age at onset (median, years) & 36 & 23 & 62 & 10 \\
Follow-up after onset (median, years) & 16 & 22 & 14 & 36 \\
With pain (\%) & 95 & 100 & 48 & 91 \\
Surgery for pain (\%) & 57 & 62 & 27 & 20 \\
Late stage CP documented by: & 86 & 86 & 80 & 91 \\
$\quad$ Calcification (\%) & 96 & 86 & 91 & 100 \\
$\quad$ Exocrine insufficiency (\%) & 66 & 33 & 41 & 73 \\
$\quad$ Diabetes (\%) & & & & \\
Summary of a personal series & & & &
\end{tabular}

$\mathrm{CP}$, chronic pancreatitis; ACP, alcoholic chronic pancreatitis; IJCP, idiopathic juvenile chronic pancreatitis; ISCP, idiopathic senile chronic pancreatitis; HP, hereditary chronic pancreatitis

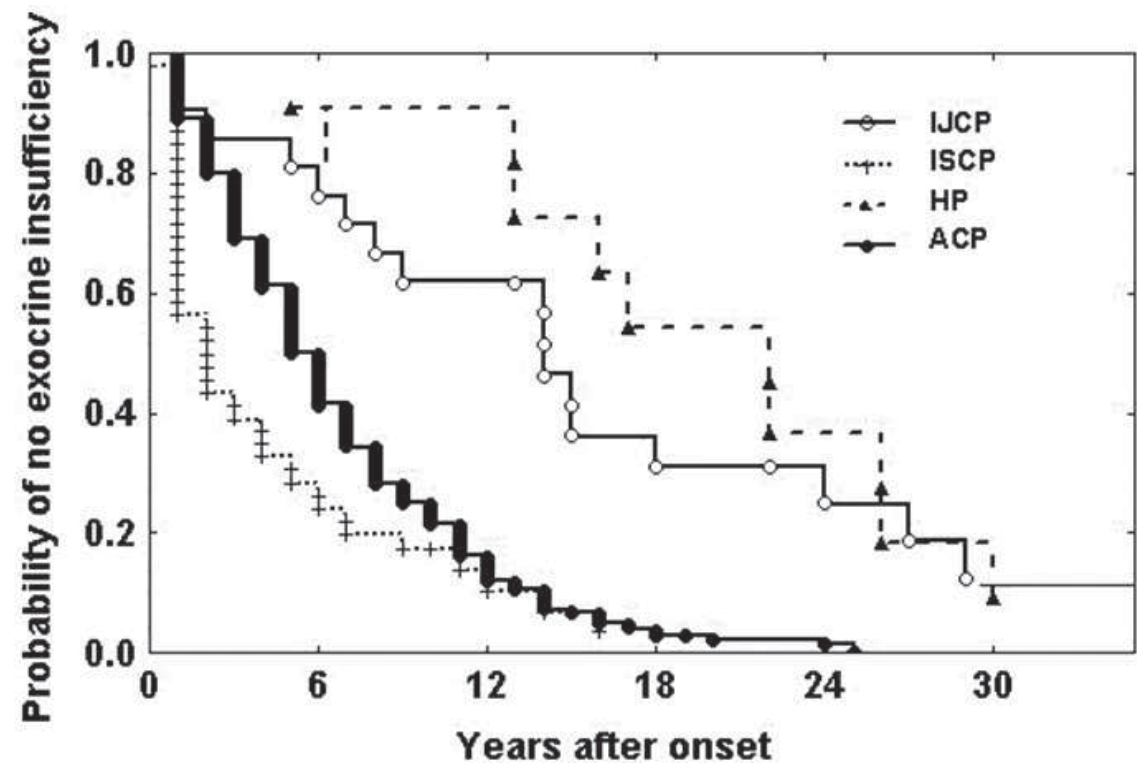

Fig. 9. The cumulative rate of exocrine insufficiency is significantly delayed after onset in nonalcoholic $\mathrm{CP}$, that is, hereditary $(H P)$ and idiopathic juvenile (IJCP) $\mathrm{CP}$, compared with alcoholic $\mathrm{CP}(A C P)$ (Kaplan-Meier method). In idiopathic senile CP (ISCP) without pain (about $50 \%$ of patients with ISCP), exocrine insufficiency was observed at onset/diagnosis in $70 \%$ of cases (modified data from reference 7 ) 

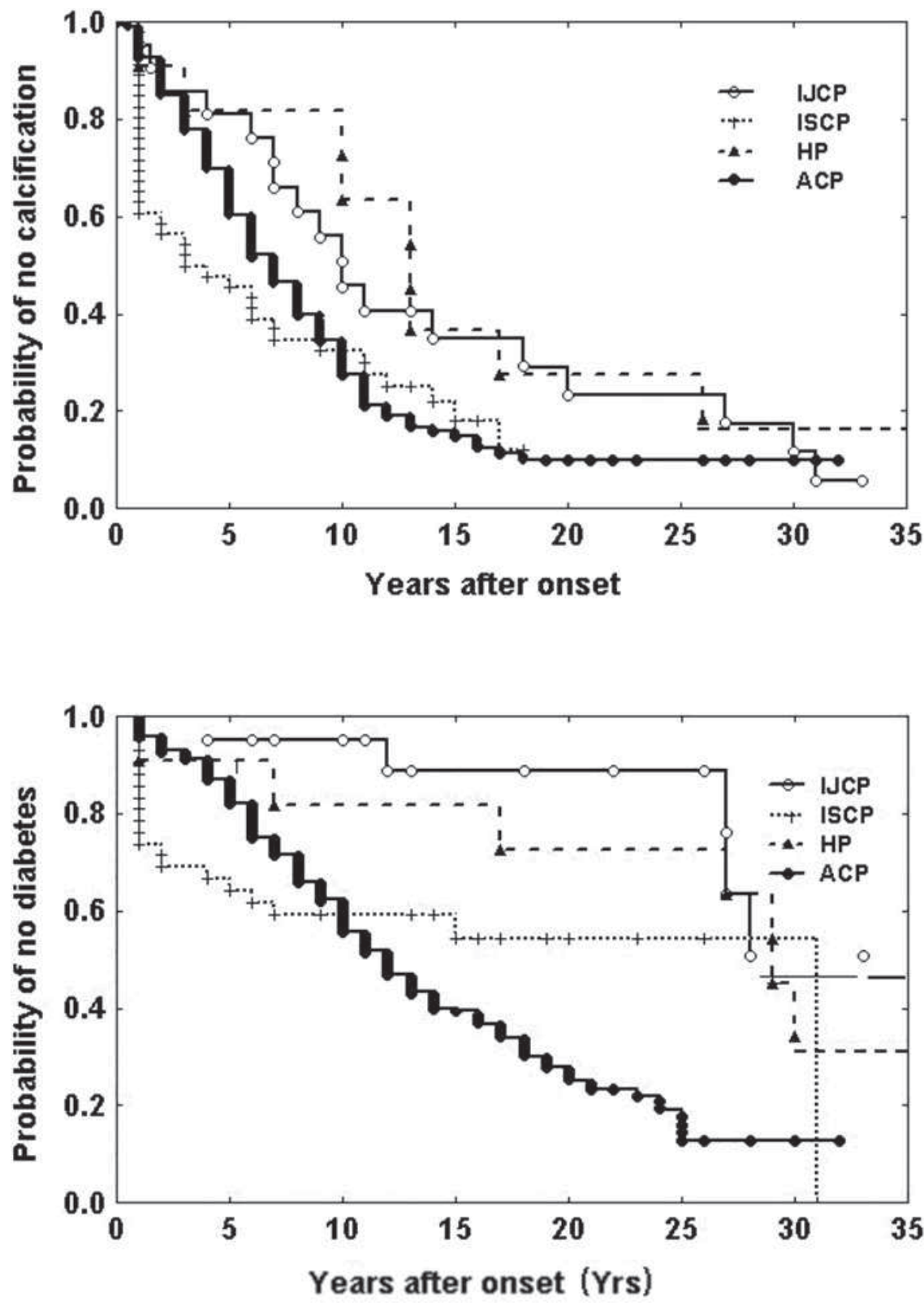

Fig. 10. Probability of remaining free of calcification after onset (see Fig. 9). Calcification developed with a marked delay after onset in HP and IJCP compared with ACP; the $50 \%$ calcification rate in ACP/ISCP was 3 to 7 years after onset compared with 11 to 14 years in HP/IJCP (no statistical difference) (modified data from reference 7)
Fig. 11. Probability of remaining free of diabetes from onset (see Fig. 9). Differences were statistically significant between HP/IJCP and ACP/ISCP. In primary painless ISCP, 12 patients $(50 \%)$ had diabetes at onset/diagnosis (modified data from reference 7)

\section{Conclusions}

1. $\mathrm{CP}$ is a dynamic long-term evolving process, which may be divided chronologically into two stages: early-stage and late-stage CP.

2. Late-stage CP can be diagnosed reliably by the markers of CP: calcification, marked ductal changes, and progressive exocrine (endocrine) insufficiency. The diagnostic criteria are identical for both alcoholic and nonalcoholic late-stage CP.

3. Clinically, early-stage $\mathrm{CP}$ is characterized by recurrent acute pancreatitis, except in primary painless $\mathrm{CP}-\mathrm{a}$ condition rare in alcoholic but frequent in nonalcoholic CP, particularly ISCP (50\%).
4. The diagnostic gold standard of early-stage CP is histology (rarely available).

5. Acute gallstone pancreatitis, in contrast to $\mathrm{ACP}$, usually does not progress to $\mathrm{CP}$.

6. A time lag, averaging 4.5-5.5 years, occurs between acute onset alcoholic pancreatitis and documentation of late-stage CP.

7. The rate of progression from early- to late-stage $\mathrm{CP}$ varies considerably for several not clearly defined reasons, perhaps in relation to continued alcohol abuse and smoking.

8. Etiology has a major impact on the progression rate of CP; i.e. marked delay in development of exocrine insufficiency and calcification after disease onset in 
HP and IJCP compared to ACP. Therefore, diagnosis of CP may be markedly delayed in nonalcoholic compared with alcoholic CP.

9. A precise definition of etiologically different types (i.e., classification) and of different stages of pancreatitis (i.e., staging) is necessary for an improved understanding among experts. At present, terms such as acute and chronic pancreatitis are used with different meanings by different authors.

\section{References}

1. Ammann RW. A clinically based classification system for alcoholic chronic pancreatitis: summary of an international workshop of experts. Pancreas 1997;14:215-21.

2. Klöppel G, Maillet B. Pathology of acute and chronic pancreatitis. Pancreas 1993;8:659-70.

3. Comfort MW, Gambill EE, Baggenstoss AH. Chronic relapsing pancreatitis: a study of 29 cases without associated disease of the biliary or gastrointestinal tract. Gastroenterology 1946;6:239-85, 376-408.

4. Gullo L, Priori P, Labo G. Natural history of acute pancreatitis and its relationship to chronic pancreatitis. In: Banks PA, Porro GB, editors. Acute pancreatitis. Milan: Masson Italia Editori; 1984. p. 87-93.

5. Ammann RW, Akovbiantz A, Largiadèr F, Schueler G. Course and outcome of chronic pancreatitis. Gastroenterology 1984;82: $820-8$.

6. Layer P, Yamamoto H, Kalthoff L, Clain JE, Bakken LJ, DiMagno EP. The different courses of early- and late-onset idiopathic and alcoholic chronic pancreatitis. Gastroenterology 1994;107:1481-7.
7. Mullhaupt B, Truninger K, Ammann RW. Impact of etiology on the early stage of chronic pancreatitis: a long-term prospective study. Z Gastroenterol 2005;43:1293-301.

8. Gullo L, Barbara L, Labo G. Effect of cessation of alcohol use on the course of pancreatic dysfunction in alcoholic pancreatitis. Gastroenterology 1988;95:1063-8.

9. Maisonneuve P, Lowenfels AB, Mullhaupt B, Cavallini G, Lankisch PG, Andersen JR, et al. Cigarette smoking accelerates progression of alcoholic chronic pancreatitis. Gut 2005;54:510-4.

10. Ammann RW, Heitz PhU, Klöppel G. Course of alcoholic chronic pancreatitis: a prospective clinicomorphological long-term study. Gastroenterology 1996;111:224-31.

11. Lankisch PG. Natural course of chronic pancreatitis. Pancreatology 2001;1:3-14.

12. DiMagno EP. Gene mutations and idiopathic chronic pancreatitis: clinical implications and testing. Gastroenterology 2001;121: 1508-12.

13. Etemad B, Whitcomb DC. Chronic pancreatitis: diagnosis, classification and new genetic developments. Gastroenterology 2001; 120:682-707.

14. Howes N, Lerch MM, Greenhalf W Stocken DD, Ellis I, Simon $\mathrm{P}$, et al. Clinical and genetic characteristics of hereditary pancreatitis in Europe. Clin Gastroenterol Hepatol 2004;2:252-61.

15. Ammann RW, Mullhaupt B; Zurich Pancreatitis Study Group. The natural history of pain in alcoholic chronic pancreatitis. Gastroenterology 1999;116:1132-40.

16. Girdwood AH, Marks IN, Bornman PC, Kottler RE, Cohen M. Does progressive pancreatic insufficiency limit pain in calcific pancreatitis with duct stricture or continued alcohol insult? J Clin Gastroenterol 1981;3:241-5.

17. Ammann RW. Diagnosis and management of chronic pancreatitis: current knowledge. Swiss Med Wkly 2006;136:166-74.

18. DiMagno EP. Toward understanding (and management) of painful chronic pancreatitis. Gastroenterology 1999;116:1252-7. 